\title{
Obtaining high quality transcriptome data from formalin-fixed, paraffin-embedded diagnostic prostate tumor specimens
}

\author{
Liesel M. FitzGerald ${ }^{1,2}$. Chol-hee Jung $\mathbb{1}^{3}$. Ee Ming Wong ${ }^{4,5}$. JiHoon E. Joo ${ }^{4,5}$ • Jodee A. Gould ${ }^{6}$. Vivien Vasic ${ }^{6}$. \\ Julie K. Bassett ${ }^{1} \cdot$ Neil O'Callaghan $^{4} \cdot$ Tim Nottle $^{7} \cdot$ John Pedersen $^{7}$ - Graham G. Giles ${ }^{1,8} \cdot$ Melissa C. Southey ${ }^{1,4,5}$
}

Received: 16 August 2017 / Revised: 23 October 2017 / Accepted: 24 October 2017

(c) United States \& Canadian Academy of Pathology 2018

\begin{abstract}
Prognostic genomic biomarkers that can be measured at diagnosis to aid choice of treatment options are unavailable for most common cancers. This is due in part to the poor quality and quantity of available diagnostic specimens for discovery research and to limitations in genomic technologies. Recent technical advances now enable high-density molecular analyses using suboptimal biological specimens. Here we describe the optimization of a transcriptome-specific protocol for use with formalin-fixed, paraffin-embedded (FFPE) diagnostic prostate cancer ( $\mathrm{PrCa}$ ) specimens. We applied the Ion AmpliSeq Transcriptome Human Gene Expression Kit (AmpliSeq Kit) to RNA samples extracted from 36 tumor-enriched and 16 adjacent normal tissues $\left(\mathrm{ADJ}_{\mathrm{NT}}\right)$ from 37 FFPE PrCa specimens over a series of eight pilot studies, incorporating protocol modifications from Pilots 2 to 5. Data quality were measured by (1) the total number of mapped reads; (2) the percentage of reads that mapped to AmpliSeq target regions (OnTarget\%); (3) the percentage of genes on the AmpliSeq panel with a read count $\geq 10$ (TargetsDetected\%); and (4) comparing the gene read-count distribution of the prostate tissue samples with the median gene read-count distribution of cell line-derived RNA samples. Modifications incorporated into Pilot study 5 provided gene expression data equivalent to cell line-derived RNA samples. These modifications included the use of freshly cut slides for macrodissection; increased tissue section thickness $(8 \mu \mathrm{m})$; RNA extraction using the RecoverAll Total Nucleic Acid Isolation Kit for FFPE (ThermoFisher); 18 target amplification cycles; and processing six samples per Ion PI chip. This protocol will facilitate the discovery of prognostic biomarkers for cancer by allowing researchers to exploit previously underutilized diagnostic FFPE specimens.
\end{abstract}

Many common cancers, including breast and prostate cancer, lead to a significant number of deaths per annum yet

Chol-hee Jung and Ee Ming Wong contributed equally to this study.

Electronic supplementary material The online version of this article (https://doi.org/10.1038/s41374-017-0001-8) contains supplementary material, which is available to authorized users.

Melissa C. Southey

msouthey@unimelb.edu.au

1 Cancer Epidemiology and Intelligence Division, Cancer Council Victoria, Melbourne, VIC, Australia

2 Menzies Institute for Medical Research, University of Tasmania, Hobart, TAS, Australia

3 Melbourne Bioinformatics, The University of Melbourne, Parkville, VIC, Australia

4 Department of Pathology, The University of Melbourne, Parkville, VIC, Australia prognostic biomarkers that can be measured at diagnosis remain limited [1-6]. In prostate cancer $(\mathrm{PrCa})$, the search for prognostic biomarkers using mRNA expression profiling has predominantly been based on tumor specimens obtained via radical prostatectomy (RP) [7-9] or limited to candidate gene studies $[1,10,11]$. Ideally, a prognostic signature should be identified agnostically using material taken from diagnostic tumor tissue, which in the case of

5 Precision Medicine, School of Clinical Sciences at Monash Health, Monash University, Clayton, VIC, Australia

6 Monash Health Translation Precinct, Medical Genomics Facility, Hudson Institute of Medical Research, Clayton, VIC, Australia

7 TissuPath Specialist Pathology, Mount Waverley, VIC, Australia

8 Centre for Epidemiology and Biostatistics, School of Global and Population Health, The University of Melbourne, Melbourne, VIC, Australia 
$\mathrm{PrCa}$ is usually a needle biopsy fixed in formalin and embedded in paraffin (FFPE). Until recently, mRNA expression profiling involving these specimens has been limited due to the low quantity and poor quality of RNA available from this type of sample [12].

Next-generation technologies can now be successfully applied to diagnostic FFPE material. These advances could facilitate the identification of a set of biomarkers that predict patient outcomes and inform treatment decisions. A recent study by Knudsen et al. analyzed 26 diagnostic PrCa FFPE biopsy specimens using the Affymetrix Human Exon 1.0 ST GeneChip [13] and demonstrated a high correlation between transcriptomic features in biopsies and matched RP specimens $(r=0.96)$. Here we describe the optimization and application of another gene expression platform, the Ion AmpliSeq $^{\mathrm{TM}}$ Transcriptome Human Gene Expression Kit (AmpliSeq $^{\text {TM }}$ Kit; ThermoFisher Scientific), to 52 diagnostic PrCa FFPE specimens.

\section{Materials and methods}

\section{Study cohorts}

Tumor material for this study was identified from five epidemiological studies led by the Cancer Council Victoria, four of which have been described in detail previously: the Melbourne Collaborative Cohort Study (MCCS) [14, 15]; the Risk Factors For Prostate Cancer Case-Control Study (RFPCS) [16, 17]; the Early Onset Prostate Cancer Family Study (EOPCFS) [18]; and the Radical Prostatectomy Registry [19]. The Aggressive Prostate Cancer (APC) study consists of Victorian men diagnosed with prostate cancer between 2 January 2010 and 25 January 2013 and agematched, PSA-screened and biopsy negative controls. Eligible cases were men diagnosed with high-grade tumors; i.e., Gleason score $\geq 7(4+3)$ and/or $\geq \mathrm{T} 3$ stage. Cases from all five studies were followed passively from date of diagnosis via linkage to the Victorian Registry of Births, Deaths, and Marriages, and the National Death Index, both of which obtained cause of death data from the Australian Bureau of Statistics. For this study, follow-up ended 31 March 2013, which was the latest date for which complete cause of death information was available at the time of specimen selection. Written informed consent was obtained from each participant and the study was approved by the Human Research Ethics Committee of the Cancer Council Victoria.

\section{Study specimens}

This study included pathology specimens from 22 MCCS cases, 11 RFPCS cases, 11 EOPCFS cases, four RPR cases, and four APC cases. For each case, FFPE diagnostic pathology material (a transrectal ultrasound (TRUS)-guided biopsy or transurethral resection (TURP) of the prostate) was retrieved from the diagnostic service laboratory and reviewed by an experienced pathologist (JP). For 14 $(26.9 \%)$ cases, representative haematoxylin and eosin (H\&E)-stained slides and unstained sections $(3 \mu \mathrm{m})$ had been prepared and stored desiccated at $4{ }^{\circ} \mathrm{C}$ for up to 15 years. For the remaining cases $(n=38)$, H\&E slides and unstained sections $(8 \mu \mathrm{m})$ were cut immediately prior to RNA extraction.

\section{RNA extraction from FFPE prostate tumor material}

Tumor areas representative of the overall Gleason score and adjacent benign areas were identified by a pathologist (JP and $\mathrm{TN}$ ) and marked directly on the representative H\&Estained section. On average, five $3 \mu \mathrm{m}$ (previously cut) or 8 $\mu \mathrm{m}$ (freshly cut) unstained sections were deparaffinized using a standard xylene and ethanol procedure prior to macrodissection of prostate tumor and adjacent histologically normal tissue $\left(\mathrm{ADJ}_{\mathrm{NT}}\right)$. RNA was extracted using the RNeasy FFPE Kit (QIAGEN) or the RecoverAll ${ }^{\mathrm{TM}}$ Total Nucleic Acid Isolation Kit for FFPE (ThermoFisher Scientific) as per the manufacturer's instructions except that RNA was eluted in a final volume of $30 \mu$ of nuclease-free water and stored at $-80^{\circ} \mathrm{C}$. The quantity and quality of FFPE RNA was measured using the Agilent 2100 Bioanalyzer system (RNA 6000 Pico Kit) according to the manufacturer's instructions (Table 1).

\section{The Ion AmpliSeq ${ }^{\mathrm{Tm}}$ Transcriptome Human Gene Expression Kit}

Gene expression was evaluated using the AmpliSeq ${ }^{\mathrm{TM}}$ Transcriptome Human Gene Expression Kit (ThermoFisher Scientific). Complementary DNA (cDNA) was generated with SuperScript ${ }^{\circledR}$ VILO $^{\mathrm{TM}}$ cDNA Synthesis Kit using 10-110 ng of FFPE RNA or $4 \mathrm{ng}$ of total RNA (Table 1) following by library preparation using Ion AmpliSeq technology and the human gene expression core panel to generate barcoded libraries. Libraries were quantified by qPCR using the Ion Library Quantification Kit, diluted to $100 \mathrm{pM}$ and pooled equally, with either eight or six individual samples per pool. Each chip contained at least one lymphoblastic cell line-derived RNA sample as a positive control. In addition, each pilot study contained at least one replicate prostate tissue sample with 25 samples repeated across the eight pilots (Table 1). Pooled libraries were amplified using emulsion PCR on an Ion Torrent OneTouch2 instrument and enriched following the manufacturer's instructions. Libraries were then loaded onto an Ion P1 chip V2 and sequenced on the Ion Torrent Proton ${ }^{\mathrm{TM}}$ sequencing system, using the Ion PI sequencing 200 Kit v3 chemistry. 


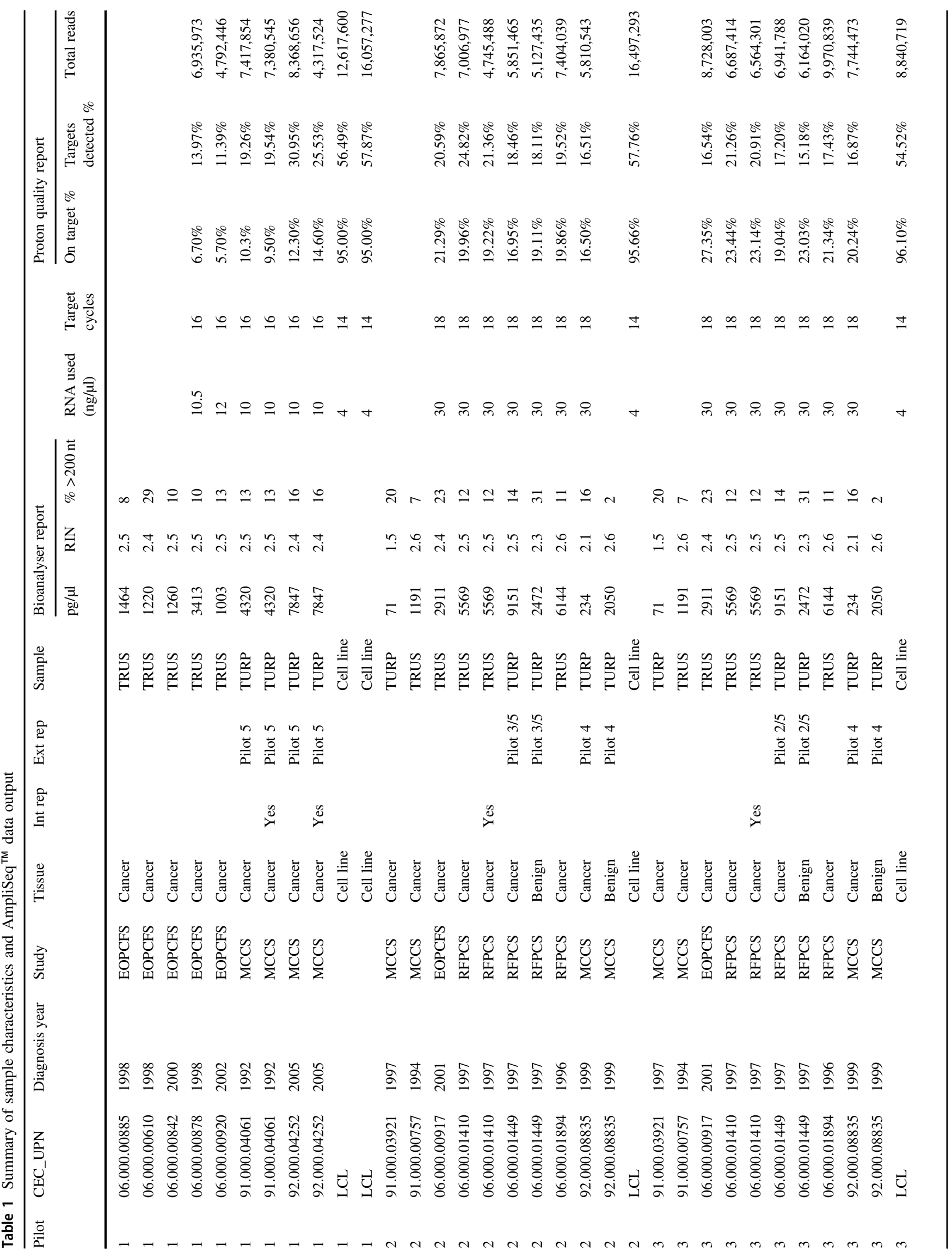




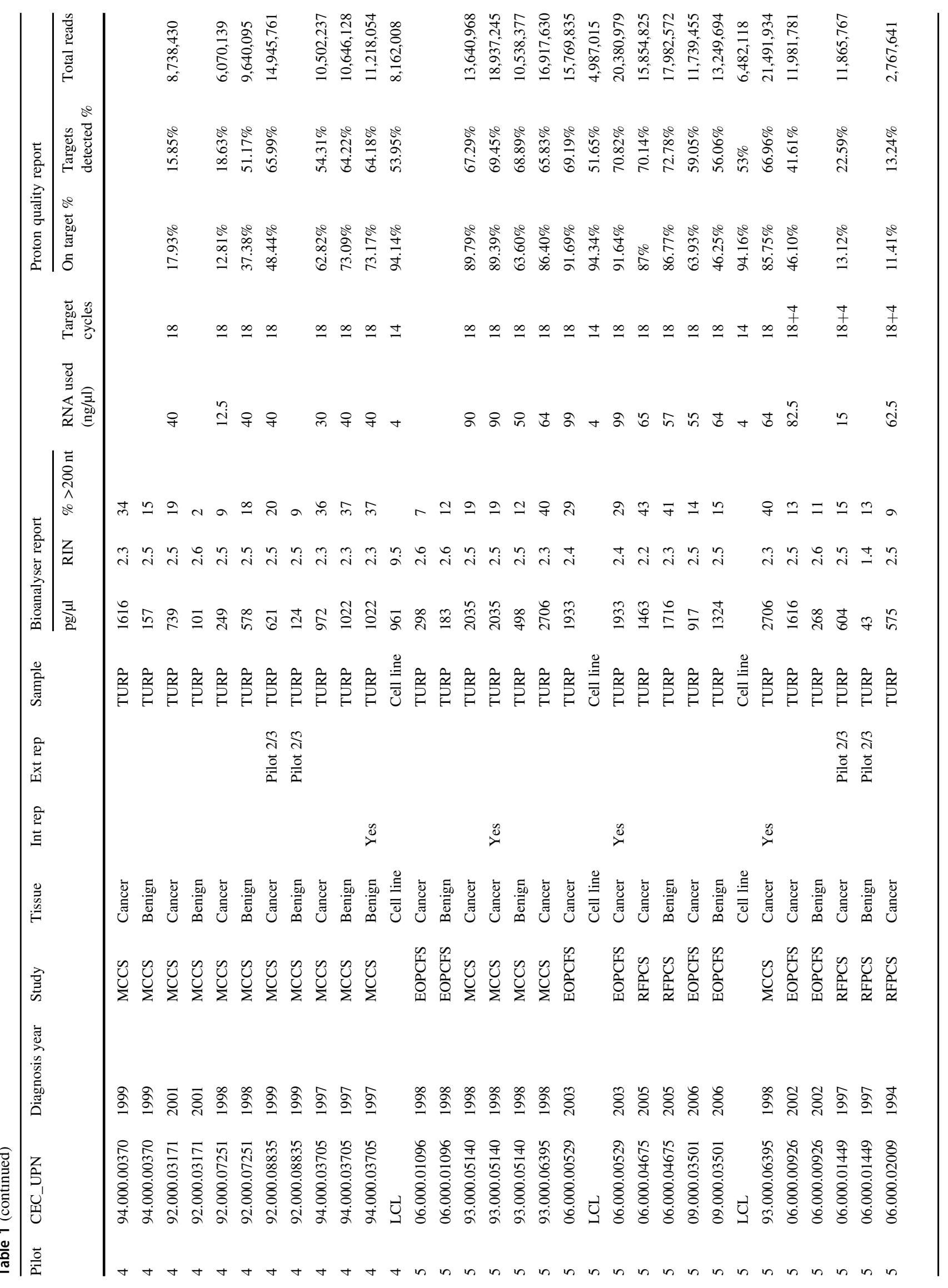




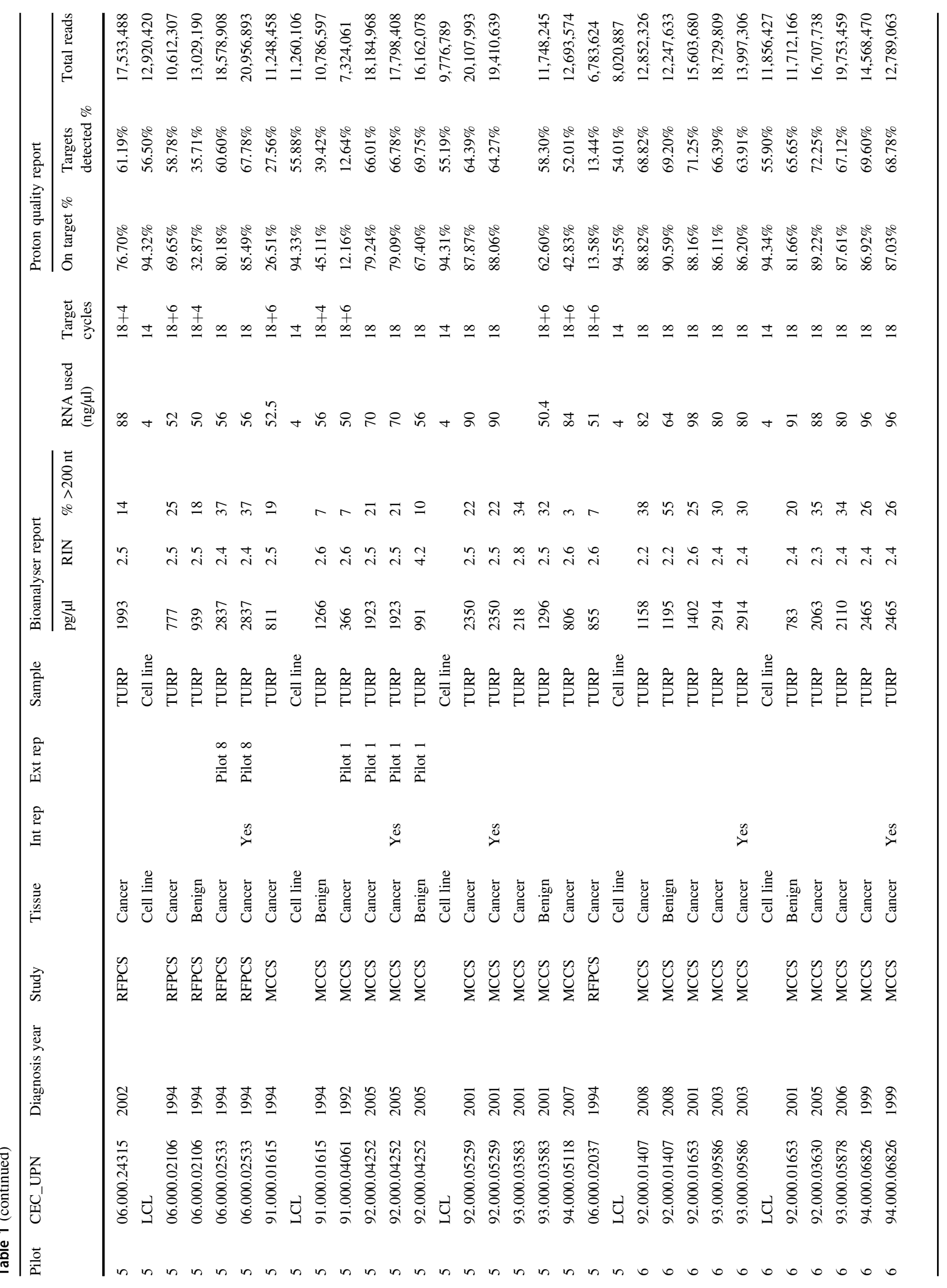




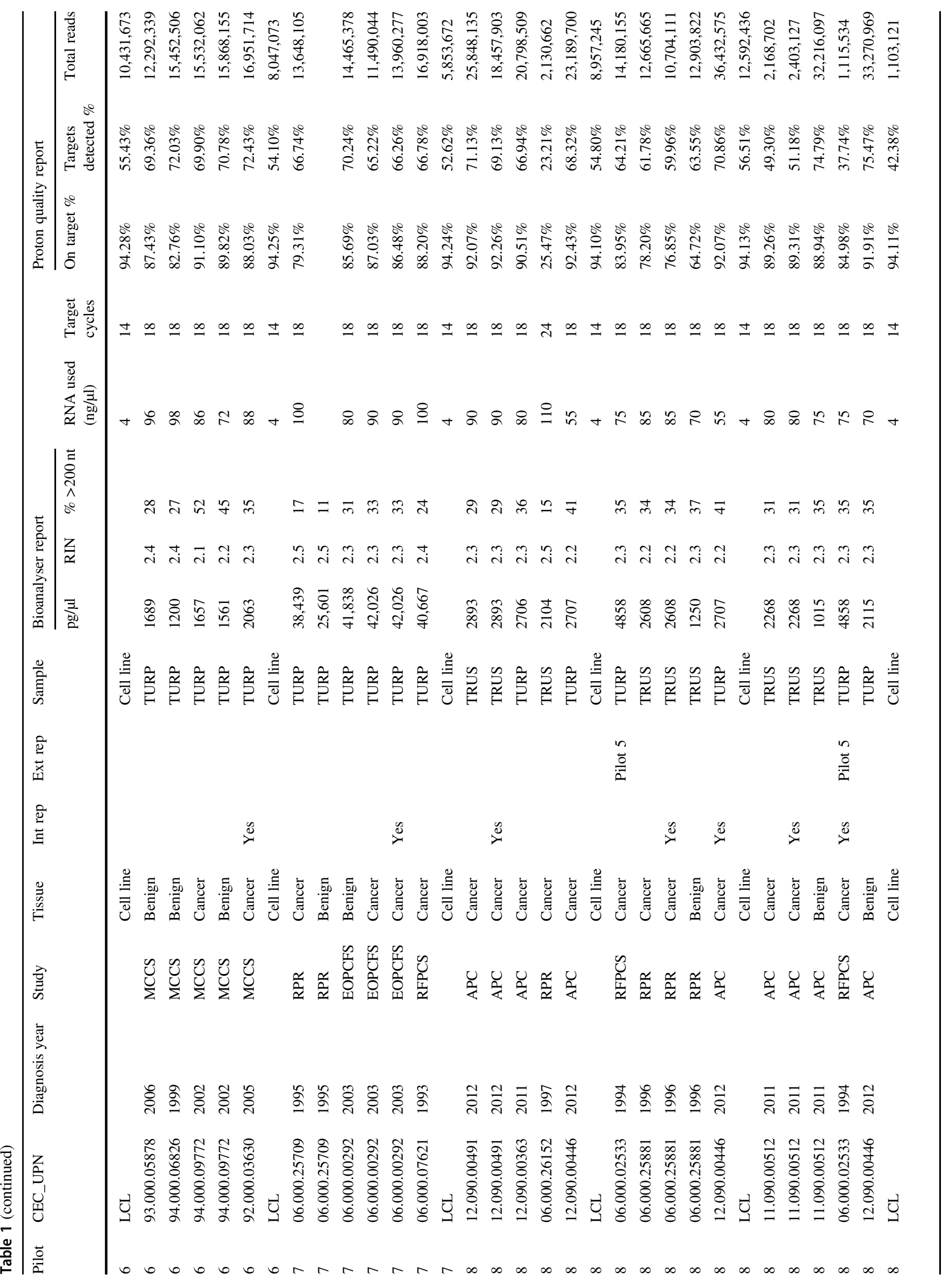




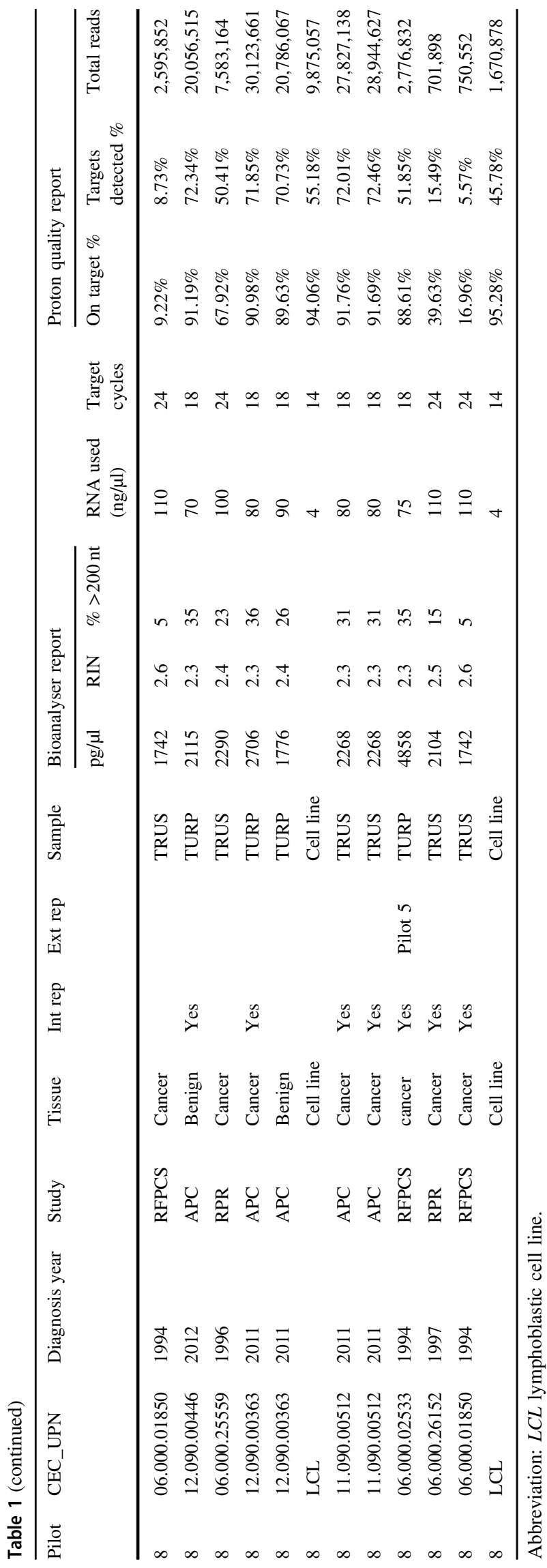

\section{Protocol optimization}

The first pilot utilized $3 \mu \mathrm{m}$ tumor tissue slides, which had been stored at $4{ }^{\circ} \mathrm{C}$, and run according to the standard manufacturer's instructions for the AmpliSeq ${ }^{\mathrm{TM}}$ Kit. For each successive pilot, protocol modifications, such as cDNA synthesis time, starting input concentrations and amplification cycles, were made with the aim of optimizing the quality of the sequencing output data (Table 2).

\section{Data analysis}

The Ion Torrent Suite v5.02 software was used to map reads. Raw read-count data files were imported into the $\mathrm{R}$ environment v3.3.2 and edgeR [20] was used for read-count normalization and differential gene expression (DGE) analysis.

The quality of data output from each sample was assessed according to the (1) total number of mapped reads; (2) percentage of reads that mapped to AmpliSeq target regions (OnTarget\%); (3) percentage of genes on the Ion AmpliSeq panel that had a read count $\geq 10$ (TargetsDetected\%); and (4) comparison of the read-count distribution, where the gene read count of the prostate tissue samples is compared with the median gene readcount distribution of the control cell line-derived RNA samples. The read-count distribution was calculated for each sample by examining the fraction of genes on the Ion AmpliSeq panel $(20,802$ target genes) within each readcount interval: $[0-1),\left[1-2^{2}\right),\left[2^{2}-2^{3}\right), \ldots,\left[2^{19}-2^{20}\right)$. The median gene read-count distribution of cell line-derived RNA samples was determined by the median of each interval across the 20 samples. After filtering for goodquality samples, the raw read counts of replicate samples were merged.

\section{Results}

\section{Sample summary}

Diagnostic FFPE tumor tissue was available for $52 \mathrm{PrCa}$ cases (TRUS $n=15$; TURP $n=37$ ), 26 of which had regions of both tumor and $\mathrm{ADJ}_{\mathrm{NT}}$ marked for RNA extraction (Table 1). After RNA extraction, 14 samples $\left(\mathrm{ADJ}_{\mathrm{NT}} n=6\right.$; cancer $\left.n=8\right)$ were not suitable for further analysis due to a small quantity and/or poor quality RNA ( $<20 \%$ of RNA fragments $>200$ nucleotides) (Table 1). In total, 44 tumor and $20 \mathrm{ADJ}_{\mathrm{NT}}$ samples $\left(19\right.$ cancer- $-\mathrm{ADJ}_{\mathrm{NT}}$ pairs) from 45 patients were processed over the series of eight pilot studies. 
Table 2 Summary of protocol changes implemented through Pilots 2-5

\begin{tabular}{|c|c|c|c|c|c|}
\hline & Pilot 1 & Pilot 2 & Pilot 3 & Pilot 4 & Pilots 5-8 \\
\hline \multirow[t]{2}{*}{ Specimen storage } & $3 \mu \mathrm{m}$ cut sections & & & $8 \mu \mathrm{m}$ cut sections & $8 \mu \mathrm{m}$ cut sections \\
\hline & $4^{\circ} \mathrm{C}$ for $\sim 10$ years & & & Freshly cut sections & Freshly cut sections \\
\hline RNA extraction & $\begin{array}{l}\text { Qiagen RNeasy } \\
\text { FFPE Kit }\end{array}$ & & $\begin{array}{l}\text { RecoverAll Total Nucleic Acid } \\
\text { Kit }\end{array}$ & $\begin{array}{l}\text { RecoverAll Total Nucleic } \\
\text { Acid Kit }\end{array}$ & $\begin{array}{l}\text { RecoverAll Total } \\
\text { Nucleic Acid Kit }\end{array}$ \\
\hline Total RNA used & $10-12 \mathrm{ng}$ & $\uparrow$ to $30 \mathrm{ng}$ & & $12.5-40 \mathrm{ng}$ & $\uparrow$ to $\geq 50 \mathrm{ng}$ \\
\hline \multirow[t]{3}{*}{ Library Prep } & AmpliSeq protocol & $\begin{array}{l}\text { Target cycles } \uparrow \\
\text { to } 18 \times\end{array}$ & Target cycles $\uparrow$ to $18 \times$ & Target cycles $\uparrow$ to $18 \times$ & $\begin{array}{l}\text { Target cycles } \uparrow \text { to } \\
18 \times\end{array}$ \\
\hline & & & Amplification cycles $\uparrow$ to $10 \times$ & & $\begin{array}{l}6 \text { samples/proton } \\
\text { chip }\end{array}$ \\
\hline & & & $\begin{array}{l}\text { Size selected for libraries } \\
(150-250 \mathrm{bp})\end{array}$ & & \\
\hline
\end{tabular}

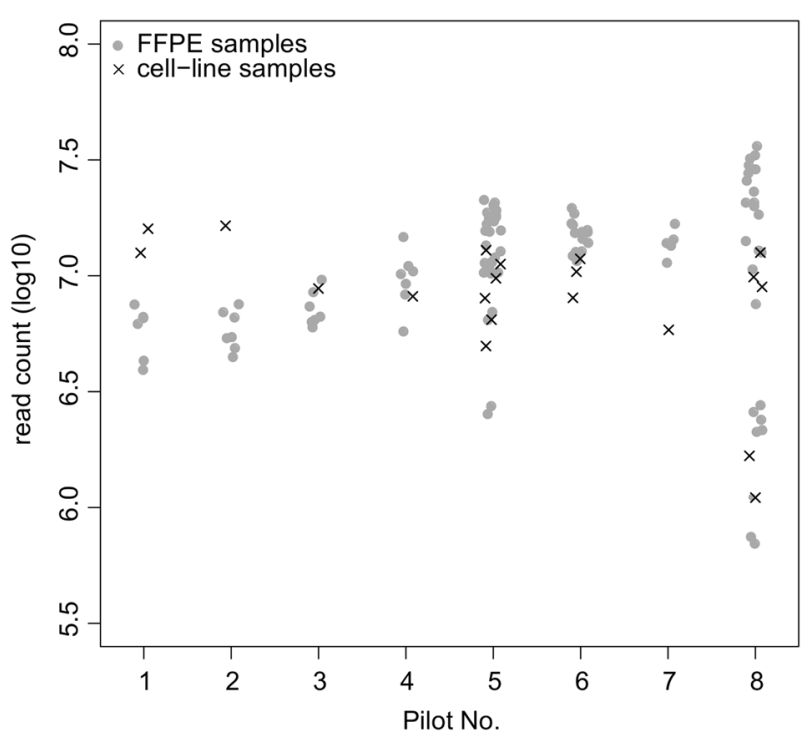

Fig. 1 Number of mapped reads for FFPE prostate tissue and cell linederived RNA samples across each of the eight pilot runs

\section{Protocol optimization}

The eight pilot studies consisted of between one and six Ion PI V2 chips (Table 1). As outlined in Table 2, several protocol changes were implemented targeting both the sample preparation and the Ion AmpliSeq ${ }^{\mathrm{TM}}$ Kit protocol. Progressive modifications to Pilots $2-5$ were assessed using individual sample measures of the total number of mapped reads, percent of reads mapped to Ion AmpliSeq target regions (OnTarget\%), and percent of Ion AmpliSeq target genes with $\geq 10$ read counts (TargetsDetected\%), and a comparison of the read-count distribution. Figure 1 shows the total number of mapped reads per sample, which increased steadily with the protocol modifications (Table 2). The OnTarget $\%$ and TargetsDetected $\%$ also improved with subsequent protocol modifications, with notable increases in both measurements from Pilot 5 onward (Fig. 2). All prostate tissue samples in Pilots 1-3 had OnTarget\% and TargetsDetected $\%$ below $40 \%$, but these measures increased from Pilot 4 onward, with the majority of prostate tissue samples having $>60 \%$ OnTarget $\%$ reads and $>50 \%$ TargetsDetected\% in Pilots 5-8. Four samples performed particularly poorly in Pilot $8(<40 \%$ OnTarget\% reads); these were TRUS samples (two replicate pairs) that had a comparatively low percentage of RNA template $>200$ nucleotides in length compared with other Pilot 8 samples (Table 1).

The gene read-count distribution was calculated for each of the prostate tissue samples and compared with the median read-count distribution of the 20 cell line-derived RNA samples (Fig. 3; full results-Supplementary Fig. 1). A high correlation between prostate and cell line-derived RNA read-count distributions was observed when prostate tissue samples reached $>40 \%$ of OnTarget $\%$ reads, and a similar result was observed for TargetsDetected\% (Supplementary Fig. 2). These results demonstrate that the protocol changes incorporated at Pilot 5 (Table 2) generally produced higher quality data that were comparable to those obtained from the cell line-derived RNA samples.

\section{Performance of replicate samples}

To further investigate the outcome of the protocol changes implemented in Pilots 1-5, we measured the correlation of gene expression profiles between replicate samples. Across the pilot runs, 25 samples (both tumor and $\mathrm{ADJ}_{\mathrm{NT}}$ ) were processed at least twice, resulting in 32 within-pilot replicates, including 16 within-chip replicates. Figure 4 shows the correlation coefficient (Pearson's) of replicates increased with successive pilots, with the majority achieving a correlation coefficient $>0.9$ from Pilot 5 onward. Apart from Pilot 1 samples, even replicates consisting of low OnTarget $\%$ and TargetsDetected $\%$ had relatively good correlation coefficient scores $(>0.8)$. This is probably due to the fact that only highly expressed genes had been amplified in the 
Fig. 2 A OnTarget $\%$ and B TargetsDetected $\%$ for FFPE prostate tissue and cell linederived RNA samples across each of the eight pilot runs. Cell line-derived RNA samples are denoted with an "X," and each chip is represented by a different color

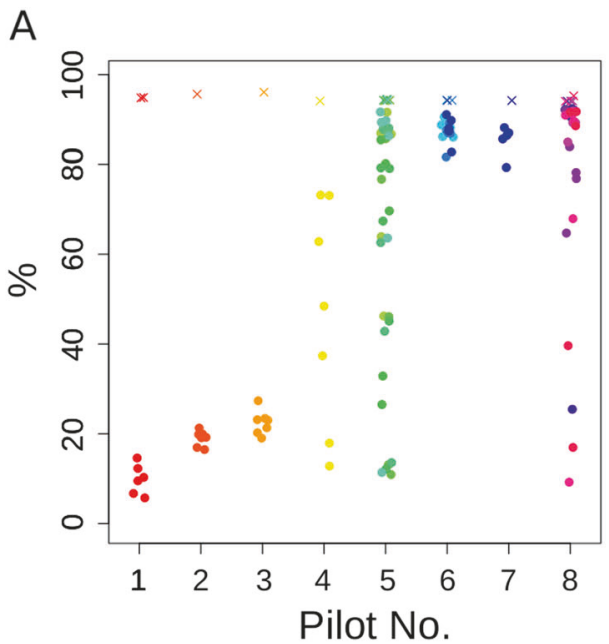

poor-quality samples, and although there were very few read counts, they still correlated.

\section{Selection of high-quality samples}

Hierarchical cluster analysis was then performed on OnTarget $\%$, TargetsDetected $\%$, and the correlation coefficient between the read-count distributions of the cell linederived RNA and prostate tissue samples (Fig. 5). Poorquality samples, which had low-quality output for all three measures, clustered separately from two other groups; the first consisting of samples with high-quality output for all three measures, and the second consisting of moderately performing samples with variable outputs across the three quality measures. Based on the hierarchical clustering, samples of higher quality were selected using the following high stringency cutoffs: OnTarget $\%>60 \%$; TargetsDetected $\%>50 \%$; and read-count correlation coefficient $>0.75$ (Supplementary Fig. 3). Using these criteria, 64 samples were classified as high-quality and the majority of these were processed in Pilots 5-8. This optimized protocol is outlined in Fig. 6. For a less stringent selection of high-quality samples, a single criterion of an OnTarget\% $>50 \%$ could be applied, as even with lower mapped reads and read-count correlation coefficients, these samples clustered with the samples defined as high-quality using the more stringent criteria (Supplementary Fig. 4).

\section{TRUS vs. TURP specimens}

The majority of samples used in this study were TURP specimens $(n=78 ; 75.7 \%)$, with TRUS specimens $(n=25$; $24.3 \%$ ) processed in the earlier pilots $(1,2,3)$ and in Pilot 8. Fifteen TRUS samples (including six replicates) processed using the optimal protocol in Pilot 8 were classified as highquality based on the more stringent criteria. The four TRUS samples that were classified as poor-quality had comparatively low percentages of RNA fragments $>200$ nucleotides (5-15\%). The fifth TRUS sample was classified as moderate quality but had a comparatively average percentage of total RNA fragments $>200$ nucleotides (31\%), and its replicate sample was classified as high-quality. The only other samples that were not scored as high quality from Pilot 5 onward were ten TURP specimens in Pilot 5 that had $<20 \%$ of RNA fragments $>200$ nucleotides. Notably, the highquality TRUS samples from Pilot 8 had a similar distribution of OnTarget $\%$, TargetsDetected $\%$, and read-count correlation measures as the high-quality TURP specimens (Supplementary Fig. 5). Of the high-quality TRUS samples, the cores ranged in length from 17 to $34 \mathrm{~mm}$ and the extracted lengths from 5.8 to $17 \mathrm{~mm}$ (further information available in Supplementary Table 1).

\section{Analysis of high-quality samples}

The data set defined as high quality included 16 samples that were replicated at least once. Hierarchical cluster analysis resulted in replicate pairs clustering tightly, with the exception of one sample, 06.000.02533 (Supplementary Fig. 6). To further examine this sample, Pearson's correlation coefficients were calculated for each of the 06.000.02533 replicate pairs and indicated a high correlation of data $(>0.8 ; p<0.001)$. Based on these results, we merged the read counts of all replicate samples, which resulted in a final high-quality data set consisting of 41 samples.

To further investigate our high-quality PrCa data set in the context of known differentially expressed genes (DEGs) and results from The Cancer Genome Atlas (TCGA), we performed analysis on TMM normalized data from the 15 $\mathrm{ADJ}_{\mathrm{NT}}$ and 26 prostate tumor samples. This analysis identified 2630 DEGs (FDR <0.01), of which 1275 were 
Fig. 3 Gene read-count distribution of FFPE prostate tissue samples compared to the median read-count distribution of the 20 high-quality, control cell line-derived RNA samples (gray dotted lines). A FFPE prostate tissue samples with an OnTarget\% < 40. B-D FFPE prostate tissue samples with an OnTarget $\%>40 \%$. The correlation between FFPE prostate tissue and cell linederived RNA sample read-count distributions was measured using Pearson's correlation coefficient
A
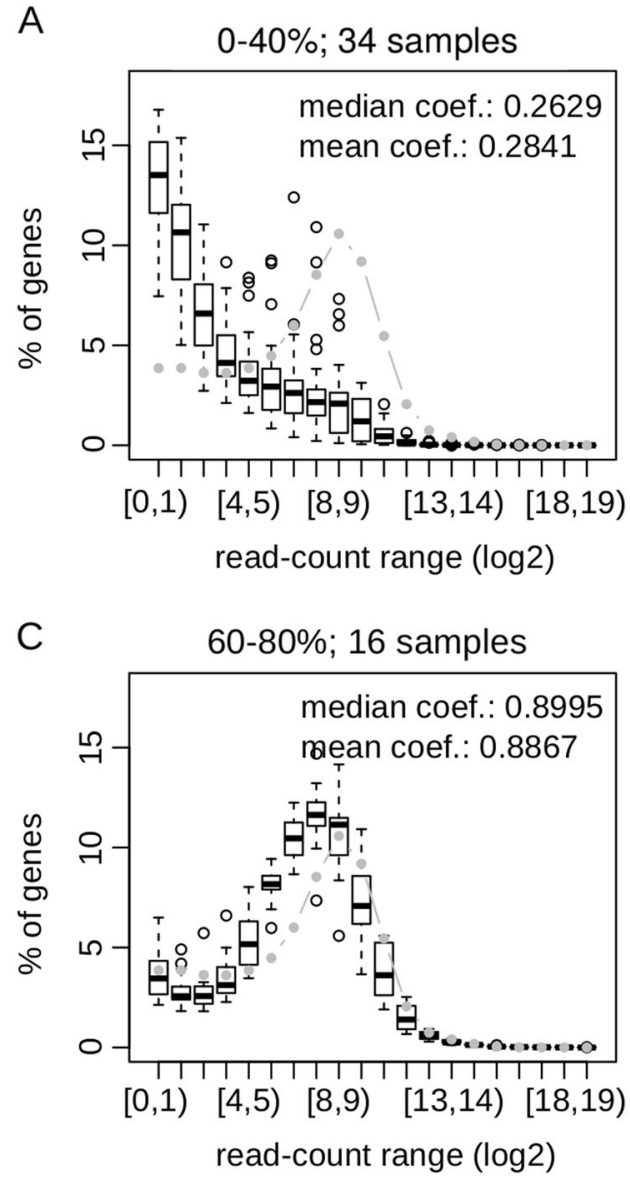

B

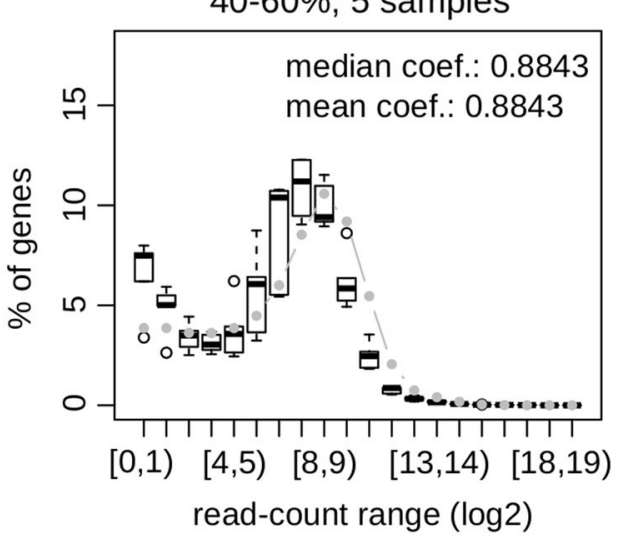

D

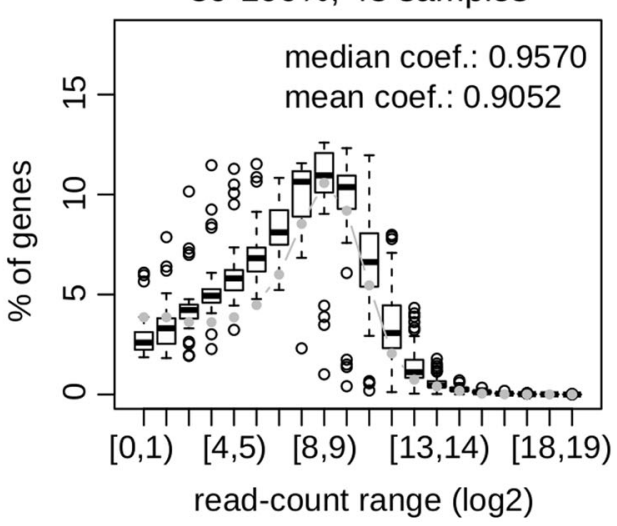

upregulated and 1355 were downregulated (Fig. 7). The top five DEGs included ADRAIA, HIF3A, PGM5, PGM5P2, and $R R N 3 P 2$, which were downregulated in tumor compared with $\mathrm{ADJ}_{\mathrm{NT}}$ (Fig. 8a). These five genes are also downregulated in tumor compared with benign tissue in TCGA (data not shown). In addition, several other genes known to be differentially expressed in tumor and $\mathrm{ADJ}_{\mathrm{NT}}$ were identified within our set of 2630 significantly DEGs, including AMACR [21], MYC [22, 23], AOX1 [24], SCGB3Al [24], and HPN [25, 26] (Fig. 8b).

\section{Discussion}

We have demonstrated that RNA derived from archival FFPE diagnostic prostate tissue can be successfully utilized for gene expression profiling using the Ion AmpliSeq Transcriptome Human Gene Expression Kit. Adopting several changes to the protocol, we were able to generate high-quality data from FFPE prostate cancer tissue that was equivalent to cell line-derived RNA.

Our final protocol included several changes to the original workflow, including the use of freshly cut slides for macrodissection; increased tissue section thickness of $8 \mu \mathrm{m}$;
RNA extraction using the RecoverAll Total Nucleic Acid Isolation Kit for FFPE (ThermoFisher); 18 target amplification cycles; and processing a maximum of six samples per chip. Using data generated from both the prostate tissue and positive control cell line, we determined that a high-quality sample (appropriate for DGE analysis) was defined by an OnTarget $\%>60 \%$, a TargetsDetected $\%>50 \%$, and a readcount correlation coefficient $>0.75$. Additionally, we have found that samples should have at least $20 \%$ of RNA fragments with $>200$ nucleotides in length before library preparation.

This study has shown that if FFPE specimen slides are freshly sectioned, high-quality gene expression data can be obtained from small amounts of starting tissue (e.g., TRUS prostate specimens), including that from FFPE tissue blocks that have been stored for over a decade. Specimens were collected and processed in several pathology laboratories across the state of Victoria, Australia, from 1992 to 2012. Over this period, collection, fixation, and processing of TRUS and TURP specimens has remained relatively consistent, whereby specimens are placed immediately after collection in buffered formalin and fixed for a maximum of $8 \mathrm{~h}$. Some variation in specimen processing within and between the laboratories is likely given the range 
Fig. 4 A comparison of gene expression profiles between replicate samples measured using Pearson's correlation coefficient. The correlation coefficient was calculated for replicate pairs processed $\mathbf{A}$ on the same chip and $\mathbf{B}$ within the same pilot

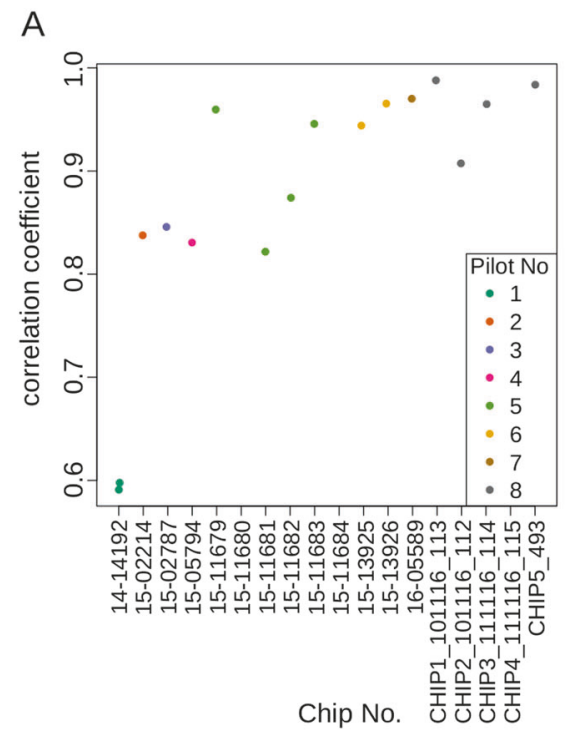

B

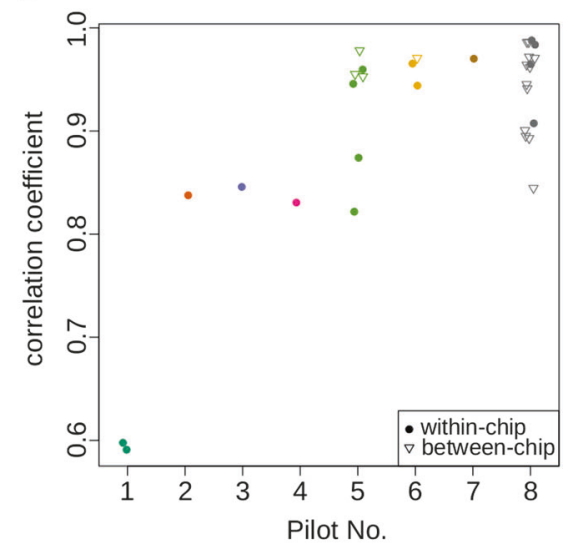

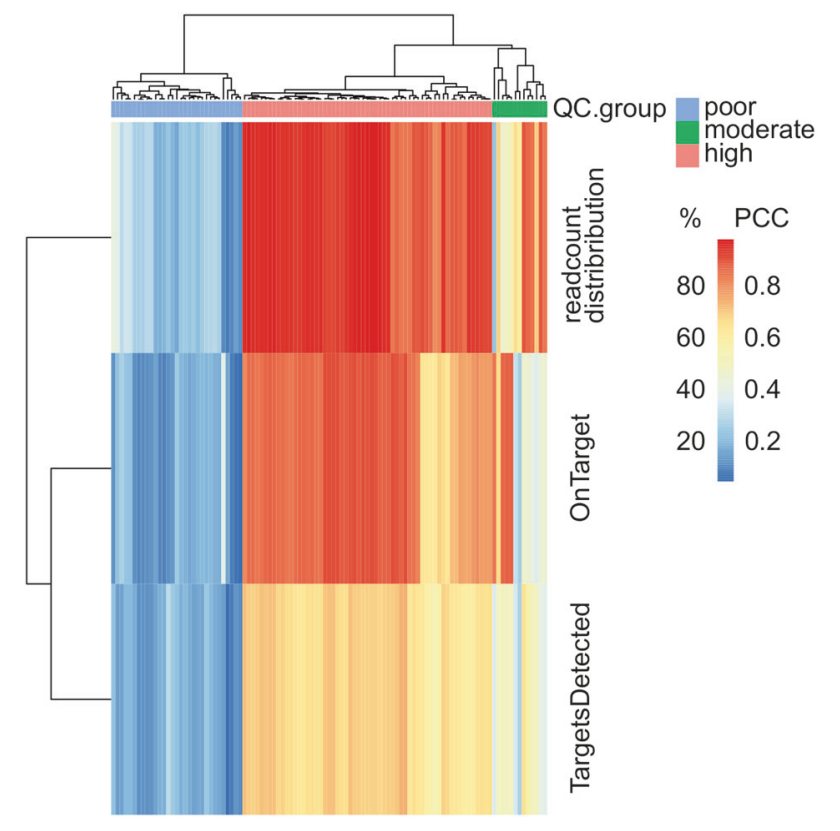

Fig. 5 Clustering of FFPE prostate tissue samples based on the quality metrics OnTarget $\%$, TargetsDetected\%, and the Pearson's correlation coefficient (PCC) between the read-count distributions of the FFPE prostate tissue and control cell line-derived RNA samples

of settings from which these specimens were collected; however, our study also demonstrates that the optimized AmpliSeq protocol is robust to any variations that may have been present over this period of time. Although our study was based on diagnostic PrCa tissue specimens, our protocol using the AmpliSeq Kit can be applied to any small volume FFPE tissue specimen, thus increasing the scope of gene expression studies to cancer biopsy specimens. This is particularly pertinent to prognostic studies, which have

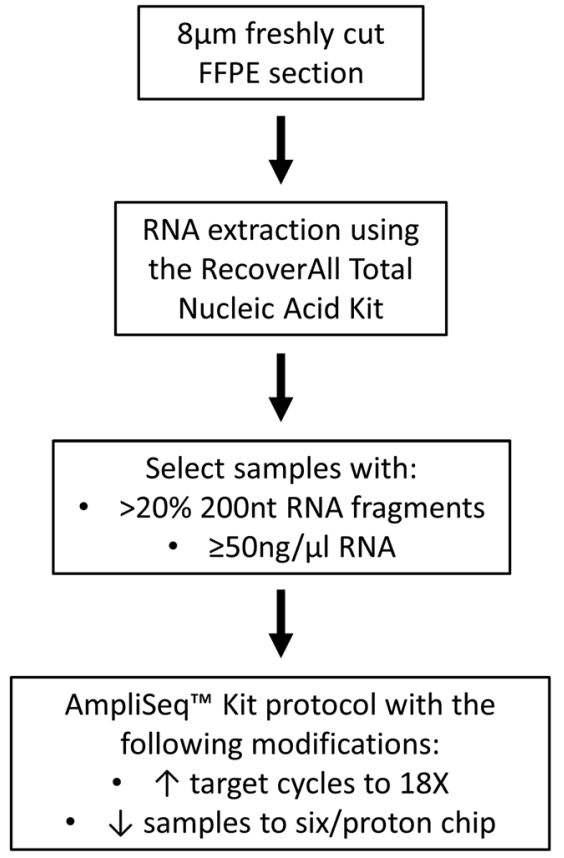

Fig. 6 Flow diagram of the final optimized protocol

previously been limited due to the quantity and quality of diagnostic FFPE biopsy samples.

To estimate the reliability of the gene expression data, we performed DGE analysis on high-quality adjacent benign and prostate tumor samples, resulting in the identification of 2630 significantly DEGs (FDR <0.001). The direction of change in gene expression for our top five DEGs, ADRA1A, $H I F 3 A$, PGM 5, PGM SP2, and RRN3P2, was the same as that seen in the 499 prostate adenocarcinomas in TCGA. Two of these genes, ADRAIA and HIF3A, have been shown to be involved in prostate cancer biology: stimulation of 
ADRAIA results in the proliferation of the $\mathrm{LNCaP}$ epithelial cell line [27] and HIF3A is hypermethylated in radical prostatectomy tumor cells $[24,28]$, resulting in reduced gene expression levels [24]. Several other genes known to be involved in prostate cancer biology (e.g., AMACR, MYC, $A O X 1, S C G B 3 A 1$, and $H P N$ ) were also found to be significantly differentially expressed in our tumor samples and in the same direction as observed by prior studies [21-26].

Recently, Knudsen et al. applied the Affymetrix Human Exon 1.0 ST GeneChip to 26 FFPE prostate cancer biopsy specimens and observed good correlations between transcriptomic features in the biopsies and matched RP specimens $(r=0.96)$ [13]. Interestingly, they observed that the

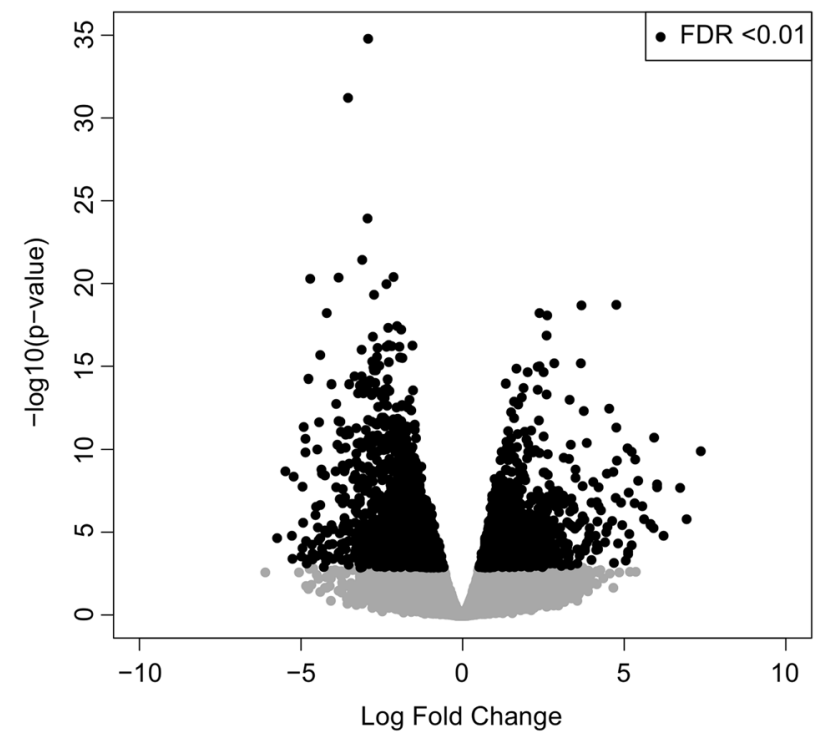

Fig. 7 Volcano plot of differential gene expression in FFPE prostate cancer vs. adjacent benign $\left(\mathrm{ADJ}_{\mathrm{NT}}\right)$ samples. Differentially expressed genes (DEGs; FDR $<0.01 ; n=2630$ ) are labeled in black correlation of a Decipher prognostic expression signature between matched specimens was not as robust $(r=0.70)$, and hierarchical clustering and PCA analysis of the data revealed two large clusters based upon the sample origin, i.e., biopsy vs. RP. These differences may be due to progression of the disease, cell transformation due to intermediate treatment, or a reflection of the tumor focus sampled and percentage of tumor epithelial cells present in the samples. One of the limitations of the study by Knudsen et al. is the variation in the amount of tumor cells in the tumor cores or punches, which ranged between $5-70 \%$ in the cores and $10-90 \%$ in the punches. In addition, some of the biopsy cores had up to $80 \%$ of stromal cells. Nonetheless, the gene expression differences between RP and biopsy samples highlight the importance of analyzing biopsy samples in order to develop a panel of prognostic markers that can be applied at diagnosis.

One of the strengths of our study is that regions of tumor and $\mathrm{ADJ}_{\mathrm{NT}}$ epithelial cells were marked by a pathologist and specific areas of interest were then macrodissected. RNA extracted from these regions were thus enriched for either tumor or $\mathrm{ADJ}_{\mathrm{NT}}$ cells, thereby reducing the proportion of stromal cells. Another strength of our design was the number of replicate samples, including several run under optimal conditions to determine the robustness of the protocol. We also included both TRUS and TURP specimens, as both form the basis of prostate cancer diagnoses. While the proportion of TRUS samples was low, the distribution of OnTarget $\%$, TargetsDetected $\%$, and read-count correlation measures were similar between high-quality TRUS and TURP specimens. In the current study, we used RNA from the lymphoblastic cell line as a data quality standard. In future studies, it would be valuable to include RNA from paired fresh frozen tumor samples, if available, as a further

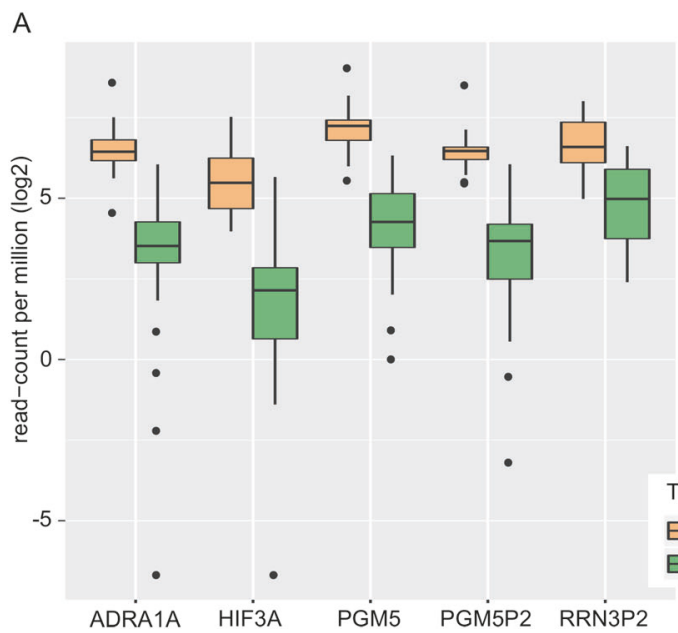

$$
\text { B }
$$

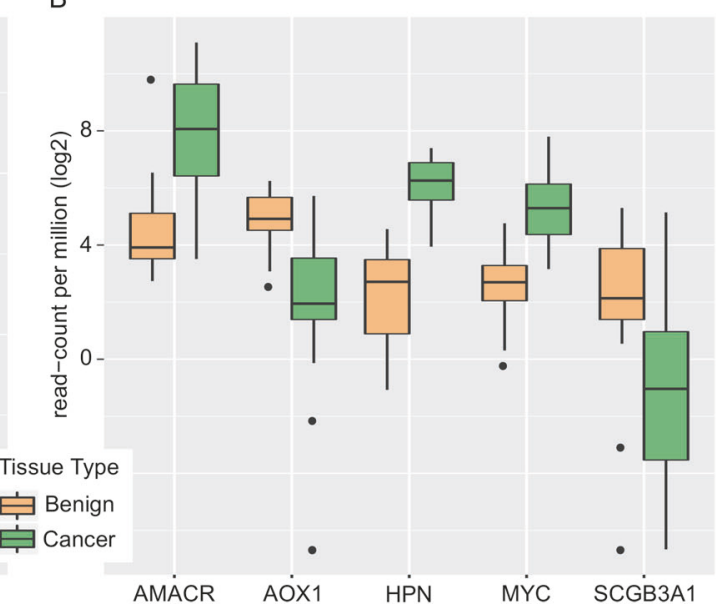

Fig. 8 Differential expression between FFPE prostate cancer and adjacent benign $\left(\mathrm{ADJ}_{\mathrm{NT}}\right)$ samples for the $\mathbf{A}$ top five most differentially expressed genes ranked by adjusted $p$ values, and $\mathbf{B}$ five genes previously shown to be differentially expressed in prostate cancer tissue 
quality standard for the FFPE specimen data and to determine the effect of formalin fixation and storage on gene expression profiles.

One of the most significant outcomes of this study, and that of Knudsen et al. [13], is the demonstration that transcriptome data can be obtained from very small quantities of FFPE tumor specimens. This has implications not only for $\mathrm{PrCa}$, but for any cancer where only small amounts of tissue are available for research. It also allows researchers to exploit stored, archival specimens that have previously been underutilized with regard to array-based gene expression studies.

Acknowledgements This work was supported by a Cure Cancer Australia/Prostate Cancer Foundation of Australia Young Investigators Grant (LMF; YI 1812). MCCS cohort recruitment was funded by VicHealth and Cancer Council Victoria. The MCCS was further supported by Australian NHMRC grants 209057, 251553, and 504711 and by infrastructure provided by Cancer Council Victoria. The RFPCS was supported by NHMRC grant 940394; the EOPCFS, and the Radical Prostatectomy Registry by Cancer Council Victoria and the APC study by NHMRC grant 623204. MCS is an NHMRC senior research fellow (1061177). We like to express our gratitude to the men who took part in the study and to the pathology laboratories in Victoria for their collaboration.

\section{Compliance with ethical standards}

Conflict of interest The authors declare that they have no conflict of interest

\section{References}

1. Cuzick J, Berney DM, Fisher G, Mesher D, Moller H, Reid JE, et al. Prognostic value of a cell cycle progression signature for prostate cancer death in a conservatively managed needle biopsy cohort. Br J Cancer. 2012;106:1095-9.

2. Gyorffy B, Bottai G, Fleischer T, Munkacsy G, Budczies J, Paladini $\mathrm{L}$, et al. Aberrant DNA methylation impacts gene expression and prognosis in breast cancer subtypes. Int J Cancer. 2016;138:87-97.

3. Jurikova M, Danihel L, Polak S, Varga I. Ki67, PCNA, and MCM proteins: markers of proliferation in the diagnosis of breast cancer. Acta Histochem. 2016;118:544-52.

4. Kazarian A, Blyuss O, Metodieva G, Gentry-Maharaj A, Ryan A, Kiseleva EM, et al. Testing breast cancer serum biomarkers for early detection and prognosis in pre-diagnosis samples. $\mathrm{Br} \mathrm{J}$ Cancer. 2017;116:501-08.

5. Leyten GH, Hessels D, Jannink SA, Smit FP, de Jong H, Cornel $\mathrm{EB}$, et al. Prospective multicentre evaluation of PCA3 and TMPRSS2-ERG gene fusions as diagnostic and prognostic urinary biomarkers for prostate cancer. Eur Urol. 2014;65:534-42.

6. McGrath S, Christidis D, Perera M, Hong SK, Manning T, Vela I, et al. Prostate cancer biomarkers: are we hitting the mark? Prostate Int. 2016;4:130-35.

7. Haldrup C, Lynnerup AS, Storebjerg TM, Vang S, Wild P, Visakorpi T, et al Large-scale evaluation of SLC18A2 in prostate cancer reveals diagnostic and prognostic biomarker potential at three molecular levels. Mol Oncol. 2016;10:825-37.

8. Penney KL, Sinnott JA, Fall K, Pawitan Y, Hoshida Y, Kraft P, et al. mRNA expression signature of Gleason grade predicts lethal prostate cancer. J Clin Oncol. 2011;29:2391-6.
9. Sinnott JA, Peisch SF, Tyekucheva S, Gerke T, Lis R, Rider JR, et al. Prognostic utility of a new mRNA expression signature of Gleason score. Clin Cancer Res. 2017;23:81-87.

10. Cooperberg MR, Simko JP, Cowan JE, Reid JE, Djalilvand A, Bhatnagar $\mathrm{S}$, et al. Validation of a cell-cycle progression gene panel to improve risk stratification in a contemporary prostatectomy cohort. J Clin Oncol. 2013;31:1428-34.

11. Klein EA, Cooperberg MR, Magi-Galluzzi C, Simko JP, Falzarano SM, Maddala T, et al. A 17 -gene assay to predict prostate cancer aggressiveness in the context of Gleason grade heterogeneity, tumor multifocality, and biopsy undersampling. Eur Urol. 2014;66:550-60.

12. Xie R, Chung JY, Ylaya K, Williams RL, Guerrero N, Nakatsuka $\mathrm{N}$, et al. Factors influencing the degradation of archival formalinfixed paraffin-embedded tissue sections. J Histochem Cytochem. 2011;59:356-65.

13. Knudsen BS, Kim HL, Erho N, Shin H, Alshalalfa M, Lam LL, et al. Application of a clinical whole-transcriptome assay for staging and prognosis of prostate cancer diagnosed in needle core biopsy specimens. J Mol Diagn. 2016;18:395-406.

14. Giles GG, English DR. The Melbourne Collaborative Cohort Study. IARC Sci Publ. 2002;156:69-70.

15. Milne RL, Fletcher AS, MacInnis RJ, Hodge AM, Hopkins AH, Bassett JK et al. Cohort profile: the Melbourne Collaborative Cohort Study (Health 2020). Int J Epidemiol. 2017;46: 1757-1757i.

16. Giles GG, Severi G, McCredie MR, English DR, Johnson W, Hopper JL, et al. Smoking and prostate cancer: findings from an Australian case-control study. Ann Oncol. 2001; 12:761-5.

17. Severi G, Hayes VM, Padilla EJ, English DR, Southey MC, Sutherland RL, et al. The common variant rs1447295 on chromosome 8q24 and prostate cancer risk: results from an Australian population-based case-control study. Cancer Epidemiol Biomark Prev. 2007;16:610-2.

18. Eeles RA, Kote-Jarai Z, Al Olama AA, Giles GG, Guy M, Severi $\mathrm{G}$, et al. Identification of seven new prostate cancer susceptibility loci through a genome-wide association study. Nat Genet. 2009;41:1116-21.

19. Bolton D, Severi G, Millar JL, Kelsall H, Davidson AJ, Smith C, et al. A whole of population-based series of radical prostatectomy in Victoria, 1995 to 2000. Aust N Z J Public Health. 2009;33:527-33.

20. Robinson MD, McCarthy DJ, Smyth GK. edgeR: a bioconductor package for differential expression analysis of digital gene expression data. Bioinformatics. 2010;26:139-40.

21. Rubin MA, Zhou M, Dhanasekaran SM, Varambally S, Barrette TR, Sanda MG, et al Alpha-methylacyl coenzyme A racemase as a tissue biomarker for prostate cancer. JAMA. 2002; 287:1662-70.

22. Hawksworth D, Ravindranath L, Chen Y, Furusato B, Sesterhenn IA, McLeod DG, et al. Overexpression of C-MYC oncogene in prostate cancer predicts biochemical recurrence. Prostate Cancer Prostatic Dis. 2010;13:311-5.

23. Nagy B, Szendroi A, Romics I. Overexpression of CD24, c-myc and phospholipase $2 \mathrm{~A}$ in prostate cancer tissue samples obtained by needle biopsy. Pathol Oncol Res. 2009;15:279-83.

24. Geybels MS, Zhao S, Wong CJ, Bibikova M, Klotzle B, Wu M, et al. Epigenomic profiling of DNA methylation in paired prostate cancer versus adjacent benign tissue. Prostate. 2015;75:1941-50.

25. Luo J, Duggan DJ, Chen Y, Sauvageot J, Ewing CM, Bittner ML, et al. Human prostate cancer and benign prostatic hyperplasia: molecular dissection by gene expression profiling. Cancer Res. 2001;61:4683-8. 
26. Xu L, Tan AC, Naiman DQ, Geman D, Winslow RL. Robust prostate cancer marker genes emerge from direct integration of inter-study microarray data. Bioinformatics. 2005;21:3905-11.

27. Thebault S, Roudbaraki M, Sydorenko V, Shuba Y, Lemonnier L, Slomianny $\mathrm{C}$, et al Alpha1-adrenergic receptors activate $\mathrm{Ca}(2+)$ - permeable cationic channels in prostate cancer epithelial cells. J Clin Invest. 2003;111:1691-701.

28. Mahapatra S, Klee EW, Young CY, Sun Z, Jimenez RE, Klee GG, et al. Global methylation profiling for risk prediction of prostate cancer. Clin Cancer Res. 2012;18:2882-95. 